\title{
Quranic Verse No. 8 of Surat Al-Jumu'ah Describes Cancer as a Complete and Accurate Description and Leads Us to Determine the True Cause of Cancer. "Part-1"
}

\author{
Mahmoud Saad Mohamed El-Khodary 1,2,3 \\ ${ }^{1}$ Department of Veterinary Medicine, Zagazig University, Zagazig, Egypt \\ ${ }^{2}$ Department of Fish Diseases Suez Canal University, Ismailia, Egypt \\ ${ }^{3}$ General Organization for Export and Import Control, Suez, Egypt \\ Email: surataljomaa@gmail.com
}

How to cite this paper: El-Khodary, M.S.M. (2018) Quranic Verse No. 8 of Surat Al-Jumu'ah Describes Cancer as a Complete and Accurate Description and Leads Us to Determine the True Cause of Cancer. "Part-1". CellBio, 7, 1-11.

https://doi.org/10.4236/cellbio.2018.71001

Received: December 1, 2017

Accepted: February 26, 2018

Published: March 1, 2018

Copyright $\odot 2018$ by author and Scientific Research Publishing Inc. This work is licensed under the Creative Commons Attribution International License (CC BY 4.0).

http://creativecommons.org/licenses/by/4.0/

\begin{abstract}
Cancer is the cell fleeing from death by blocking the pathways of the intrinsic and the extrinsic program of cell death (Apoptosis). The success depends on making the programs of cell death run again.
\end{abstract}

\section{Keywords}

Cancer Description, Intrinsic Apoptosis, Extrinsic Apoptosis

\section{Introduction}

Cancer can be defined as a disease in which a group of abnormal cells grow uncontrollably by disregarding the normal rules of cell division [1].

Cancer is an intractable disease and leads to the death of millions of people. Cancer is more dangerous than nuclear bombs. The nuclear bomb that hit Nagasaki killed 90,000 people [2]. The World Health Organization reported that in 2015, 8.8 million people around the world died from cancer. In February, 2017, cancer was responsible for almost 1 in 6 deaths globally. More than 14 million new cases are reported around the world each year and may rise to over 21 million cases by 2030 [3] [4].

For all types of treatments out there; they are unable to save all cancer patients. Cell division is not a disease, but the problem is excessive increase in the number of cells. This study determines the nature of these cells.

Somatic cells divide mitotically into two similar cells; one dies \& the other lives. This means that the normal somatic cells have two programs, division 
program and death program. The death program controls the cell numbers which are produced by division program of cell.

Cancer cells (which are somatic cells in origin) divide mitotically as well into two similar cells they both live. This means that the death program is impaired or blocked.

\section{Material \& Results}

\subsection{Programmed Cell Death (Apoptosis)}

In Greek, apoptosis translates to the "falling off" of leaves from a tree [5] [6]. In biology this term is used to mean the process of death cell that occurs in multi cellular organisms [7]. It helps the body get rid of cells it does not need. Between 50 - 70 billion cells die each day by apoptosis [8]. Apoptosis produces cell fragments called (apoptotic bodies) which are quickly removed by phagocytic cells that engulf it before the contents of cell spill out onto the surrounding cells and damage them. Once apoptosis has begun, it cannot be stopped so, it is a highly regulated process [5] [6].

Apoptosis can occur via two different pathways: Intrinsic and Extrinsic.

\subsection{Intrinsic Apoptosis}

The intrinsic pathway is activated as a result of DNA damage [9] and is controlled by the Bcl-2 family of proteins [10]. All the members of the Bcl-2 family share a close homology in up to four characteristic regions termed the (BH-domains). These $\mathrm{BH}$ domains are (BH1, $\mathrm{BH} 2, \mathrm{BH} 3$ and $\mathrm{BH} 4)$. The $\mathrm{Bcl}-2$ family is divided into pro-survival protein and pro-apoptotic proteins [11].

\subsubsection{Pro-Survival Protein}

(Anti-apoptotic protein) suppresses cell death, by controlling the mitochondrial pathway to apoptosis. Such as (Bcl-2, Bcl-Xl, Bcl-W, Mcl-1 and A1). They share $\mathrm{BH} 1, \mathrm{BH} 2$ and in some cases $\mathrm{BH} 3$ \& $\mathrm{BH} 4$ [12].

\subsubsection{Pro-Apoptosis Proteins}

These promote cell death. Pro-apoptosis proteins are divided into two categories: pro-apoptotic multi domains and pro-apoptotic one domain:

1) Pro-apoptotic multi domains: contain ( $\mathrm{BH} 1, \mathrm{BH} 2, \mathrm{BH} 3)$ as (Bak and $\mathrm{Bax}$ ). Bak is an Integral membrane protein on the outer mitochondrial surface, Whereas, Bax is a largely cytosolic protein.

2) Pro-apoptotic one domain: (BH3-only protein) as Noxa, Poma, Bim and Bad. It is a potent mediator of cell death [12].

In normal state, anti-apoptotic proteins Bcl-2 bind with pro-apoptotic proteins Bak and Bax. So, pro-apoptotic Bak and Bax are present in an inactive form. In the normal state of cell krupple-like factor occupies the promoter region of gene P53 and keeps it in an inactive form (Figure 1). But in active state (Figure 2), when DNA is damaged, the krüppel-like factor, which is bound on the promoter region of P53 gene, leaves it, resulting in activation of gene P53 


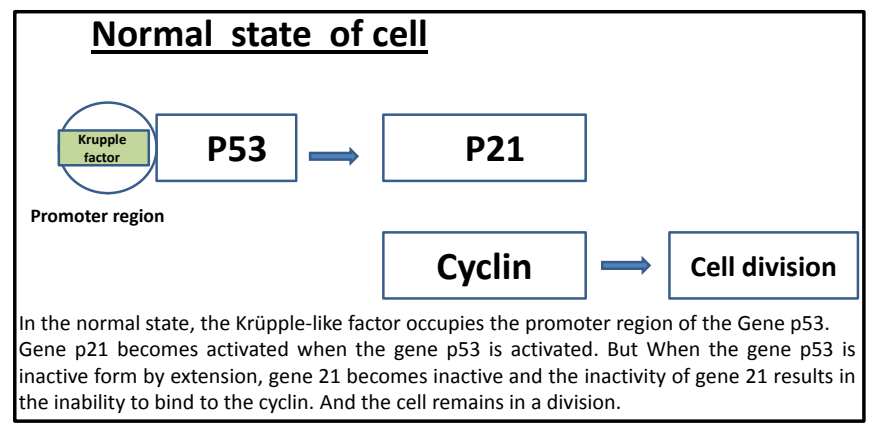

Figure 1. Shows the normal state of cell the normal state of cell.

which in turn, activates P21 (cyclin dependent-kinase inhibitor). When gene 21 is activated it binds with cyclin resulting in cell division arresting. Also the activation of gene P53 results in the production of BH3-only protein (Noxa and Puma) (Figure 2) which has direct and indirect actions:

Directly, by binding and activating Bax and Bak, forming complexes of either Puma-Noxa with Bax or Bak. These translocate to mitochondria [13].

Indirectly, by binding with anti-apoptotic proteins, including $\mathrm{Mcl}-1, \mathrm{Bcl}-2$ and Bcl-xL. This binding causes the anti-apoptotic proteins to dissociate from Bak and Bax. Bak and Bax become free [12] [14] [15] BH3-only protein binds with Bak and Bax then translocates them to the mitochondria [13]. The pro-apoptotic members Bak and/or Bax aggregate on the outer mitochondrial membrane, forming a channel called the Mitochondrial Apoptosis-Induced Channel (MAC). Once MAC formed, cytochrome-c is released from the inter-membrane space of the mitochondria to the cytosol [16] [17]. As soon as cytochrome-c is released, apoptosis cannot stop [5] [6].

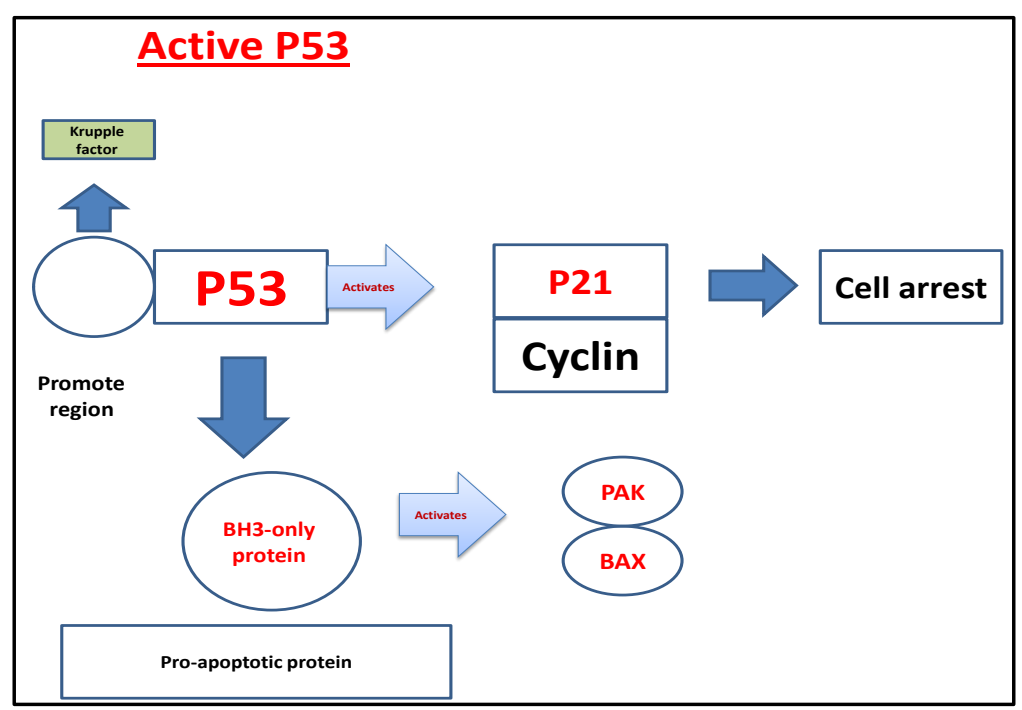

Figure 2. Shows active state of the cell when DNA is damage. The krüpple-like factor leaves the promoter region of gene P53 which leads to gene P53 activation and the subsequent activation of P21 (cyclin dependent kinase inhibitor). P21 then binds with cyclin, resulting in cyclic arrest. The activation of gene $\mathrm{P} 53$ also leads to the production of $\mathrm{BH}-3$ only protein, resulting in cell death. 


\subsection{Blocking the Pathway of the Intrinsic Program of Cell Death}

The cell flees from the intrinsic program of cell death by producing Bcl-2 and keeping gene $\mathrm{P} 53$ inactive.

The Muc1 is aberrantly over-expressed in human cancer. The Mucl is a trans membrane glycoprotein which has extracellular domain and cytoplasmic domain. The cytoplasmic domain (Mucl-c) is targeted toward the nucleus, where it interacts with P53 gene and occupies its promoter region. This makes the kruppel-like factor tightly bound with the promoter region, resulting in suppression of gene P53 [18] [19]. When gene P53 becomes inactive, this leads to suppression of P21 (cyclin dependent-kinase inhibitor) which results in prevention of cyclic arrest. This also blocks the Bh3-only protein production (Noxa and Puma) (Figure 4). Furthermore, the cytoplasmic domain of Muc-1 is associated with Bax in human cancer cells and binds directly to Bax forming Bax BH3 complex, thereby blocking Bax function, which results in repressing the mitochondrial pathway [20]. As a result, the normal cell flees from death and divides endlessly.

If we inhibit the production of Muc-1, the P53 gene becomes active again. The activation of P53 means cell cyclic arrest and the intrinsic program of cell death runs again and cancer cell dies (Figure 2). But 50\% of cancer patients have P53 gene and 50\% don't. In the case of damage to P53 gene there is no hope to make the intrinsic program of cell death run again. I remembered a verse from Quran (verse No. 8, surat $\mathrm{Al}$-jumuah).

"Say to them, the death you try to flee from will meet you; then you will be conveyed back to him who knows the unseen and the witnessed. Then he will inform you of all that you have done."

Death which you flee from will meet you. That means the death has two opposite location: behind you (from which you flee) and facing you (will meet you).

For example, I am in a room with a bomb. I will escape it by exiting the room. That way, death is behind me. But as soon as I leave the room, another bomb faces me and moves toward me. That way death is behind me (in the room) and in front of me (outside the room). This is exactly the case in normal cells: Intrinsic and Extrinsic programs of cell death.

This means that the Qur'an verse is talking about phenomenon of cell fleeing from death. The cell dies if either one of the programs of cell death runs or both but to flee from death it must flee from both programs (intrinsic \& extrinsic).

So, we must focus on the extrinsic program of cell death pathway.

\subsection{Extrinsic Apoptosis}

The activation of extrinsic program of cell death is executed by tumor necrosis factor receptors most importantly TNF receptor (TNFR) and Fas receptor (FasR). These receptors are activated by specific molecules (Ligands) which are produced by immune cells.TNF ligand (TRAIL) and Fas ligand (Fasl) activating their corresponding receptors that are present on the cells, inducing apoptosis. [21] [22]. 


\subsubsection{TNF Pathway: TNF Ligand (TRAIL)}

TNF-alpha is a cytokine produced mainly by activated macrophages and monocytes but can also be produced by many other cells including B-Lymphocytes, T-Lymphocytes and fibroblasts. TNF-alpha is the major extrinsic mediator of apoptosis [23]. Most cells in the human body have two receptors for TNF-alpha: TNFR1 and TNFR2 [24]. The interaction between TRAIL and TNFR initiates the pathway that leads to caspase enzyme activation via (FADD) Fas-Associated Death Domain and (TRADD) TNF Receptor Associated Death Domain [25].

\subsubsection{Fas Pathway}

The Fas receptor (first apoptosis signal) - Also known as Apo-1 or CD95 is a trans membrane protein of the TNF family which binds to the Fas ligand (FasL) [26]. In the absence of their ligand, death receptors are present as monomers or pre-assembled dimers or trimers on the cell surface [27] [28]. Binding of the death ligand stabilizes the death receptor in tri-meric or oligo-meric complexes and induces a conformational change leading to death receptor activation. The activated receptor complex recruits the adaptor protein FADD and initiator caspases activation (caspase-8 and/or-10) leading to the formation of the death-inducing signaling complex (DISC) [28], which contain the FADD, Caspase- 8 and Caspase-10. The caspase- 8 directly activates other members of the caspase family (Figure 3). In addition, the Fas-disc start a feedback loop that spirals into increasing release of pro apoptotic factors from the mitochondria and amplifies activation of Caspase [26] [29].

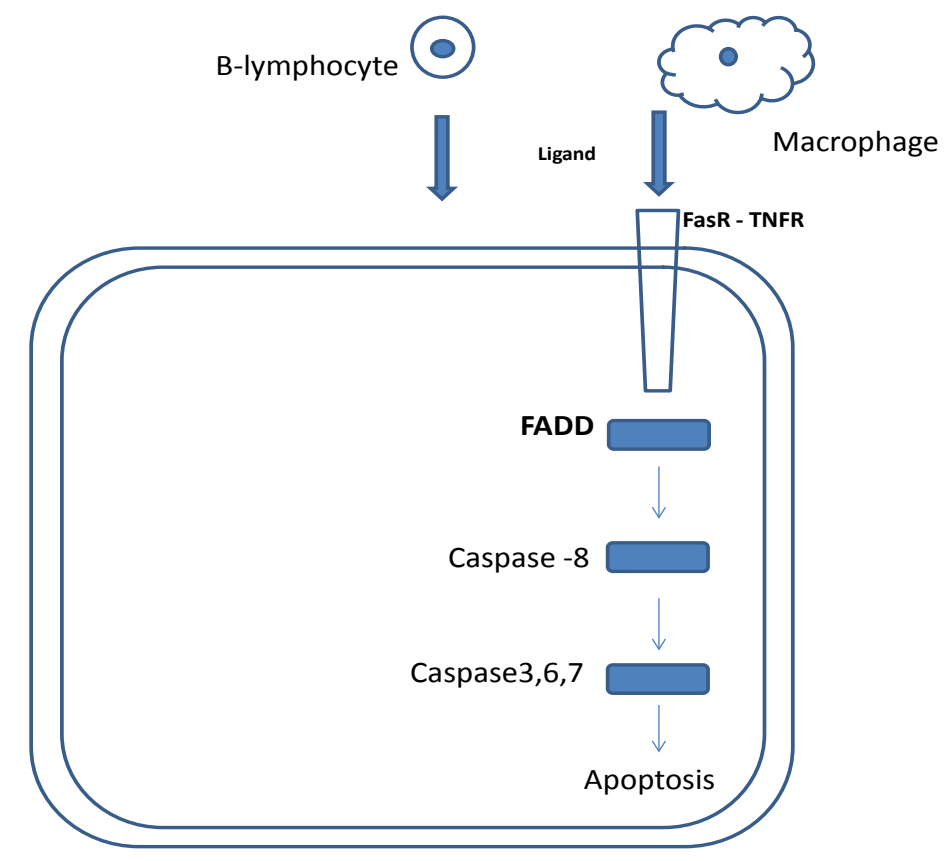

Figure 3. Shows the interaction of ligands with death receptors and activated it. The activated receptor complex recruits the adaptor protein FADD and initiator caspases activation (caspase- 8 and/or-10) leading to the formation of the death-inducing signaling complex (DISC) [28], which contain the FADD, Caspase- 8 and Caspase-10, leads to apoptosis. 


\subsection{Blocking the Pathway of the Extrinsic Program of Cell Death}

The extrinsic pathway of apoptosis depends on the interaction of ligands with their death receptors. Cells flee from extrinsic program of cell death by producing four elements, (MUC-1, MUC-4, MUC-16, MMP3 (metalloproteinase enzymes) and Decoy Receptor-3) (Figure 5).

1) MUC1:

The MUC1 cytoplasmic domain binds directly to the Fas-associated death domain (FADD) at the death effector domains, so MUC1 competes with caspase-8 for binding to FADD. So, it inhibits the extrinsic apoptosis pathway.

2) The matrix Metalloproteinase (MMPs): enzymes which cleave the ligands. So no interaction occurs.

3) Muc-16 gene which stimulates the production of mucin-16 which has long extracellular domain (tower-like) which acts like a mask on death receptors, resulting in prevention of ligands binding with the death receptors.

4) Decoy Receptor-3 (DR-3) which binds with ligands (keeps the ligands away from the death receptors). Decoy receptor 3 (DcR3) Is receptor of the necrosis factor superfamily. DcR3 is highly elevated in patients with various tumors [30]. It does not have intracellular death domain so it is unable induce apoptosis. It binds to FasL, LIGHT, and TL1A [31]. The crystal structure of FasLwas shown in complex with DcR3 [30]. It is meaning that DcR3 prevent FasR/Fasl interaction by competitively binding to membrane-bound Fas ligand and rendering them inactive.

Tumor cells can prevent Death receptors activation by inducing cleavage of death ligands from the surface of immune effector cells and FasL and TRAIL can be cleaved off the surface of immune cells by matrix metalloproteinases (MMP)-3,-7, and -9 [32] [33]. It blocks apoptosis by cleavage of death ligands (FasL and TRAIL). So, cleaving the ligands or preventing interaction with death receptor results in blocking the extrinsic pathway of program of cell death.

So, the cell flees from death by:

Blocking (Intrinsic and Extrinsic) pathways of program of cell death.

A) Blocking the intrinsic program of cell death: by suppressing the gene P53 or damaging.

Cancer cells produce Bcl-2 (anti-apoptosis protein) which binds with bax and Bak and keeps them inactive. Also, produces MUC-1 protein to prevent the activation of gene P53. this has two consequences:

1) prevention of the activation of $\mathrm{P} 21$ which prevents its binding to cyclin thus, the cells divide endlessly.

2) blocking the production of pro-apoptotic proteins $\mathrm{BH}$-only proteins (Noxa \& Puma) which prevent the activation of Bax. This means that cytochrome-c is not released into cytosol. Thus the intrinsic program of cell death is blocked.

B) Blocking extrinsic program of cell death: by expressing the production of Decoy receptor 3, Muc16, muc-4 and metalloproteinase enzymes (MMPS). 
So we can define cancer as "cell fleeing from death by blocking both intrinsic and extrinsic pathways of program of cell death".

So, the elements which are responsible for blocking the pathways of intrinsic and extrinsic programs of cell death are: 1-Muc-1, Bcl-2, Muc-4, Muc-16, Decoy receptor3 and metalloproteinase enzymes MMP3.

Blocking the pathway of intrinsic program of cell death (Figure 4).

\section{Muc-1}

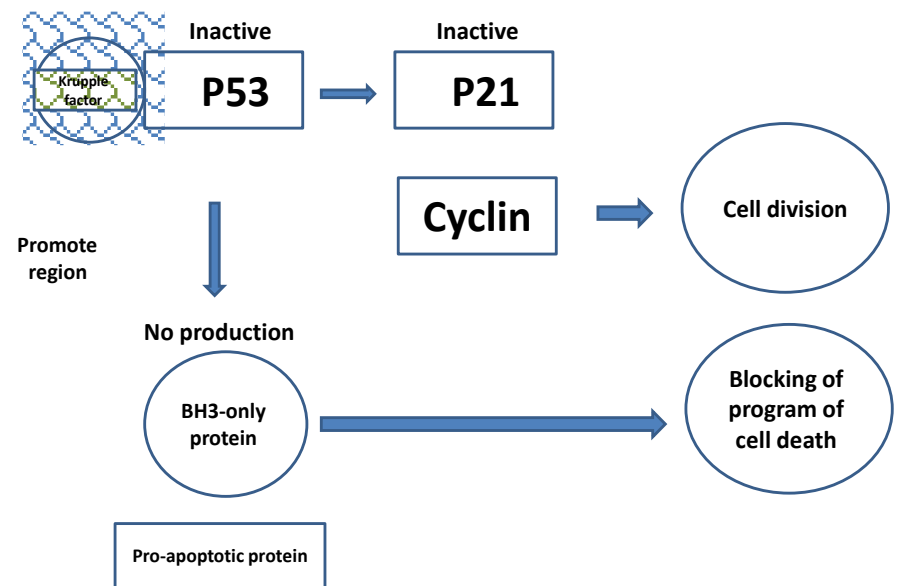

Figure 4. Shows Muc-1 interacting with gene P53 and making the krüpple-like-factor tightly bound with the promoter region, leading to the inactivation of the suppression of P21 (cyclin dependent kinase inhibitor). This results in prevention of cyclic arrest. This also blocks the $\mathrm{BH}-3$ only protein which leads to block of the intrinsic apoptosis.

Blocking the pathway of the extrinsic program of cell death (Figure 5).

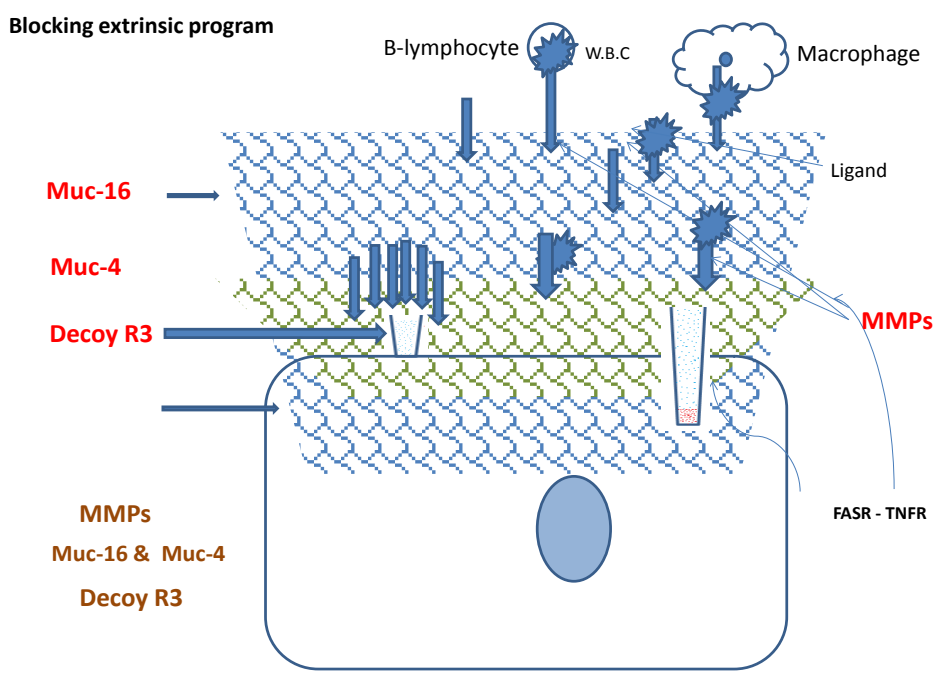

Figure 5. Shows Muc-4 and Muc-16 preventing the binding of ligands with their death receptors and acting like a mask on the death receptors. MMP3 (metalloproteinase enzymes) cleave the ligands at the surface of the immune cells and at the extracellular matrix so that no interactions occur with the death receptor. Decoy receptor-3 binds with ligands and keeps them away from their receptor. 


\section{Discussion}

The cause of cancer is a subject that has been debated widely for a long time. I believe I have found it through Allah's guidance. Let's start with the basics.

Somatic cells divide mitotically into two similar cells; one dies \& the other lives. This means that the cell has two programs; a division program \& a death program. Cancer cells (which are somatic cells in origin) divide mitotically as well into two similar cells they both also live. This means that either the death program is impaired or blocked.

The cell has two death programs: Intrinsic \& extrinsic.

Intrinsic death program:

When a cell's DNA is damaged by excessive free radicals, gene P53 is activated, which in turn activates gene P21 (cyclin dependent-kinase inhibitor). P21 binds with cyclin and stops cell division, as well as producing BH3-only protein (Noxa \& Poma). BH3-only protein activates Bax proteins and translocates them to the mitochondria causing them to aggregate on the mitochondrial surface. This result in formation of a channel that leaks cytochrome-c into cytosol. This begins the death program and cannot be stopped.

Cancer cells produce MUC-1 protein to prevent the activation of gene p53. This has two consequences:

1) Prevention: the activation of $P 21$ which prevents its binding to cyclin thus, the cells divide endlessly.

2) Blocking: the production of pro-apoptotic proteins BH3-only proteins (Noxa \& Puma) which prevent the activation of Bax. This means that cytochrome-c is not released into cytosol. Thus the intrinsic program of cell death is blocked.

Some authors activated P53-according to this research but that lead to the patient dying. So they synthesized drug called (Go-201), which removes MUC-1, but the cure rate was $50 \%$, because half the patients had lost gene P53, which means Go-201 did not work.

"Verse No. 8, surat Al-Jumah" accurately defines cancer and determines its cause. "Say to them, the death you try to flee from will meet you; then you will be conveyed back to him who knows the unseen and the witnessed. Then he will inform you of all that you have done".

When fleeing, death is behind you and upon meeting; death will be in front of you. That means the death has two opposite locations and one of them moves toward me. This applies to the cell: intrinsic \& extrinsic death program. It means that this verse is talking about "cell fleeing from death phenomenon" for the cell to flee from death; it must shut down both pathways of intrinsic and extrinsic program of cell death. That is the key of my research.

\section{Cell Flees from Intrinsic Program of Death}

When the intrinsic program of cell death is shut down, the cell divides endlessly and does not die and this is the definition of cancer. But this is not an accurate 
description, as the extrinsic program of cell death is still active and would lead to cell death. So for the cell to be truly cancerous, it must flee from the extrinsic cell death program as well. Therefore, a cancerous cell is a cell that flees from death by blocking both the intrinsic and extrinsic program of cell death. To do this, the cancer cell produces 6 elements/factors. Upon removal these of factors, the Intrinsic \& extrinsic programs run again and cells die.

Since the six elements produced by the cell to shut down the programs of intrinsic and extrinsic cell death have been recorded in all the presence of cancer cells, it means the cell produces these six elements in anticipation death and blocks intrinsic and extrinsic programs of cell death leading to:

1) The continuation of the cell in life.

2) The continuation of the cell in dividing non-stop. Where the Muc-1, which is produced by the cell, shuts down the gene P-53, which is associated with the inactivity of the 21-gene (cyclin dependent-kinase inhibitor) is not associated with the cyclin, the cell continues to divide non-stop. "This is cancer".

Secondly: The accuracy of the Quranic pronunciation of fleeing means striving and making the necessary arrangements for saving itself, which is more appropriate than the word escape.

This is proof and evidence of the accuracy of the description of the Qur'an of cancer.

Qur'an describes cellular phenomenon. The cellular phenomenon which is described by Qur'an is "cell fleeing from death", this phenomenon leads to cancer. So, we can describe cancer as:

Cancer is cells fleeing from death by blocking intrinsic and extrinsic programs of cell death.

\section{Acknowledgements}

Firstly, I wish to express sincere gratitude and deepest thanks to "ALLAH" who gave me of everything I asked, and I were to count his divine favors, I would not be able. Secondary, I would like to express my cardinal and deepest thanks to "ALLAH" who guided me, pushed me, supported me and gave me the ability and patience to read a huge number of papers and gave me a life to complete this work. I would like to thank Rasha Mohamed Mostafa, Computer Specialist, for great assistance to me in presenting this research in it is current form. I would also like to thank Shaimaa Mostafa Mohamed, young veterinarian, who has also been of great assistance to me in wording this research. I would like to thank my dear friend Dr. Reda Said Khalill for his great help in the translation of this paper and producing it in its English form.

\section{References}

[1] Hejmadi, M. (2010) Introduction to Cancer Biology. 2nd Edition. http://csbl.bmb.uga.edu/mirrors/JLU/DragonStar2017/download/introduction-to-c ancer-biology.pdf

[2] Atomic Bombings of Hiroshima and Nagasaki. 
https://en.wikipedia.org/wiki/Atomic_bombings_of_Hiroshima_and_Nagasaki

[3] WHO/Cancer-World Health Organization (2015) http://www.who.int/cancer/en/

[4] WHO (2017) WHO/World Cancer Day 2017-World-Health Organization. WHO, Geneva.

[5] Alberts, B., Johnson, A., Lewis, J., et al. (2002) Molecular Biology of the Cell. 4th Edition. Garland Science, New York.

[6] Alberts, B., Johnson, A., Lewis, J., Raff, M., Roberts, K. and Peter, W. (2008) Chapter 18 Apoptosis: Programmed Cell Death Eliminates Unwanted Cells. In: Molecular Biology of the Cell, 5th Edition, Garland Science, New York, 1115.

[7] Douglas, G. (2011) Means to an End: Apoptosis and Other Cell Death Mechanisms. Cold Spring Harbor Laboratory Press, New York.

[8] Karam and Jose A. (2009) Apoptosis in Carcinogenesis and Chemotherapy. Springer, Netherlands.

[9] Cory, S. and Adams, J.M. (2002) The BCL-2 Family: Regulators of the Cellular Life-or-Death Switch. Nature Reviews Cancer, 2, 647-656. https://doi.org/10.1038/nrc883

[10] Deng, J. (2017) How to Unleash Mitochondrial Apoptotic Blockades to Kill Cancer? Acta Pharmaceutica Sinica B, 7, No. 1. https://doi.org/10.1016/j.apsb.2016.08.005

[11] Giam, M., Hung, D.C.S., and, Bouillet, P. (2008) BH3-Only Proteins and Their Roles in Programmed Cell Death. Oncogene, 27, S128-S136. https://doi.org/10.1038/onc.2009.50

[12] Wolter, K.G., Hsu, Y.T., Smith, C.L., Nechushtan, A., Xi, X.G., and, Youle, R.J. (1997) Movement of Bax from the Cytosol to Mitochondria during Apoptosis. The Journal of Cell Biology, 139, 1281-1292. https://doi.org/10.1083/jcb.139.5.1281

[13] Vela, L., Gonzalo, O., Naval, J., and, Marzo, I. (2013) Direct Interaction of Bax and Bak Proteins with BCL-2 Homology Domain 3 (BH3)-Only Proteins in Living Cells Revealed by Fluorescence Complementation. The Journal of Biological Chemistry, 288, 4935-4946.

[14] Hsu, Y.-T., Wolter, K.G., and, Youle, R.J. (1997) Cytosol-To-Membrane Redistribution of Bax and BCL-XL during Apoptosis. Proceedings of the National Academy of Sciences, 94, 3668-3672.

[15] Rouzer, C.A. (2015) How Breast Cancer Cells Evade Death. VICB Communications, Nashville, TN.

[16] Dejean, L.M., Martinez-Caballero, S., and, Kinnally, K.W. (2006) Is MAC the Knife That Cuts Cytochrome C from Mitochondria during Apoptosis? Cell Death and Differentiation, 13, 1387-1395.

[17] Dejean, L.M., Martinez-Caballero, S., and, Kinnally, K.W. (2006) Regulation of the Mitochondrial Apoptosis-Induced Channel, MAC, by BCL-2 Family Proteins. Biochimica et Biophysica Acta, 1762, 191-201.

[18] Wei, X., Xu, H., and, Kufe, D. (2007) Human Mucin 1 Oncoprotein Represses Transcription of the $p 53$ Tumor Suppressor Gene. Cancer Research, 67, 1853-1858. https://doi.org/10.1158/0008-5472.CAN-06-3063

[19] Hollingsworth, M.A., and, Swanson, B.J. (2004) Mucin in Cancer: Protection and Control of the Cell Surface. Nature Reviews Cancer, 4, 45-60. https://doi.org/10.1038/nrc1251

[20] Ahmad, R., Alam, M., Rajabi, H., and, Kufe, D. (2012) The MUC1-C Oncoprotein Binds to the BH3 Domain of the Pro-Apoptotic Bax Protein and Blocks Bax Function. The Journal of Biological Chemistry, 287, 20866-20875. 
[21] Walczak, H. (2013) Death Receptor-Ligand Systems in Cancer, Cell Death and Inflammation. Cold Spring Harbor Perspectives in Biology, 5, a008698.

[22] Mocarski, E.S., Kaiser, W.J., Livingston-Rosanoff, D., Upton, L.W., Daley-Bauer, L.P. (2014) True Grit: Programmed Necrosis in Antiviral Host Defense, Inflammation, and Immunogenicity. The Journal of Immunology, 192, 2019-2026.

[23] Idriss, H.T., and, Naismith, J.H. (2000) TNFa and TNF Receptor Superfamily: Structure-Function Relationship(s). Microscopy Research and Technique, 50, 184-195. https://doi.org/10.1002/1097-0029(20000801)50:3<184::AID-JEMT2>3.0.CO;2-H

[24] Heller, R.A., Song, K., Fan, N., and, Chang, D.J. (1992) Tumornecrotsis Factor Receptor Mediates Cytotoxicity. Cell, 70, 47-56. https://doi.org/10.1016/0092-8674(92)90532-H

[25] Chen, G., and, Goeddel, D.V. (2002) TNF-R1 Signaling: A Beautiful Pathway. Science, 296, 1634-1635.

[26] Wajant, H. (2002) The Fas Signaling Pathway: More than a Paradigm. Science, 296, 1635-1636.

[27] Chan, F.K.-M., Chun, H.J., Zheng, L., Siegel, R.M., Bui, K.L., and, Lenardo, M.J. (2000) Adomain in TNF Receptors That Mediates Ligand-Independent Receptor Assembly and Signaling. Science, 288, 2351-2354.

https://doi.org/10.1126/science.288.5475.2351

[28] Sessler, T., Healy, S., Samali, A., and, Szegezdi, E. (2013) Structural Determinants of DISC Function: New Insights into Death Receptor-Mediated Apoptosis Signaling. Pharmacology \& Therapeutics, 140, 186-199. https://doi.org/10.1016/j.pharmthera.2013.06.009

[29] Wajant, H. (2007) Connection Map for Fas Signaling Pathway. Science Stke. 2007: $\operatorname{tr} 1$.

[30] Lau, W., Ramagopal, U., Cheng, H., Bonanno, J.B., Toro, R., Bhosle, R., Zhan, C., and, Almo, S.C. (2016) Crystal Structure of the Complex of Human FasL and Its Decoy Receptor DCR3. Structure, 24, 2016-2023.

[31] Sheikh, M.S., and, Fornace, A.J. (2000) Death and Decoy Receptors and p53-Mediated Apoptosis. Leukemia, 14, 1509-1513. https://doi.org/10.1038/sj.leu.2401865

[32] Kiaei, M., Kipiani, K., Calingasan, N.Y., Wille, E., Chen, J., Heissig, B., et al. (2007) Matrix Metalloproteinase-9 Regulates TNF- $\alpha$ and FasL Expression in Neuronal, Glial Cells and Its Absence Extends Life in a Transgenic Mouse Model of Amyotrophic Lateral Sclerosis. Experimental Neurology, 205, 74-81.

[33] Ebsen, H., Lettau, M., Kabelitz, D., and, Janssen, O. (2015) Subcellular Localization and Activation of ADAM Proteases in the Context of FasL Shedding in T Lymphocytes. Molecular Immunology, 65, 416-428. https://doi.org/10.1016/j.expneurol.2007.01.036 